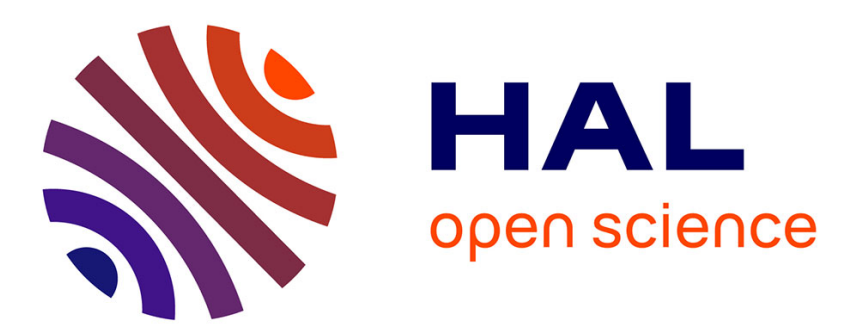

\title{
Time-varying Channel Complex Gains Estimation and ICI Suppression in OFDM Systems
}

\author{
Hussein Hijazi, Laurent Ros
}

\section{To cite this version:}

Hussein Hijazi, Laurent Ros. Time-varying Channel Complex Gains Estimation and ICI Suppression in OFDM Systems. Globecom 2007 - IEEE Global Communication Conference (Globecom'07)

(Globecom'07), Nov 2007, Washington, DC, United States. 5 p. hal-00275599

\section{HAL Id: hal-00275599 \\ https://hal.science/hal-00275599}

Submitted on 24 Apr 2008

HAL is a multi-disciplinary open access archive for the deposit and dissemination of scientific research documents, whether they are published or not. The documents may come from teaching and research institutions in France or abroad, or from public or private research centers.
L'archive ouverte pluridisciplinaire HAL, est destinée au dépôt et à la diffusion de documents scientifiques de niveau recherche, publiés ou non, émanant des établissements d'enseignement et de recherche français ou étrangers, des laboratoires publics ou privés. 


\title{
Time-varying Channel Complex Gains Estimation and ICI Suppression in OFDM Systems
}

\author{
Hussein Hijazi, and Laurent Ros \\ GIBSA-lab, CNRS INPG - BP 46 - 38402 Saint-Martin-d'Hères - France
}

\begin{abstract}
In this paper, we present an iterative algorithm for channel complex gains estimation with inter-sub-carrierinterference (ICI) reduction in orthogonal-frequency-divisionmultiplexing downlink mobile communication systems using comb-type pilot. Assuming delays information, the time-variation of the multipath complex gains within one OFDM symbol are obtained by interpolating the symbol time-average values, which have been previously estimated with LS criteria. After that, the channel matrix is computed and the ICI is reduced by using successive interference suppression (SIS) in data symbol detection. The algorithm's performance is further enhanced by an iterative procedure, performing channel estimation and ICI suppression at each iteration. Mean Square Error theoretical analysis and simulation results show a significant performance improvement for high normalised Doppler spread (especially after the first iteration) in comparison to conventional methods.
\end{abstract}

Index Terms-OFDM, ICI, SIS, channel estimation, timevarying channels.

\section{INTRODUCTION}

O RTHOGONAL frequency division multiplexing (OFDM) is widely considered as the most promising communication technique in the current broadband wireless mobile communication systems due to high robustness to the multipath interference [1]. Assuming insertion of pilot-tones (called comb-type pilot) into each OFDM symbols, the conventional channel estimation methods consist generally of estimating the channel at pilot frequencies and next interpolating the channel frequency response. Estimation of the channel at the pilot frequencies can be based on Least Square (LS) criterion, or Linear Minimum Mean-SquareError (LMMSE) criterion for better performance [2]. In [3], low-pass interpolation (LPI) has been shown to perform better than all interpolation techniques used in channel estimation.

In [4] the channel estimator is based on a parametric channel model, which consists to estimate directly the time delays and complex attenuations of the multi-path channel. This estimator yields the best performance among all comb-type pilot channel estimators, with the assumption that the channel is invariant within one OFDM symbol. However, OFDM system is vulnerable when the channel changes within one OFDM symbol. In such case, the orthogonality between subcarriers is easily broken down, resulting in inter-sub-carrier-interference (ICI) so that system performance may be considerably degraded.

In this paper, we present an iterative algorithm for channel estimation with inter-sub-carrier-interference (ICI) reduction

The authors are with the GIPSA lab. (ex LIS lab.), Grenoble, France (email: hussein.hijazi@lis.inpg.fr, laurent.ros@lis.inpg.fr.) in OFDM downlink mobile communication systems using comb-type pilot. Exploiting the nature of Radio-Frequencies channel, the delays are assumed invariant (over several OFDM symbols) and perfectly estimated, and only the complex gains of the multi-path channel have to be estimated as we have already done in CDMA context [8]. Note that an initial very performant multipath time delays estimation can be obtained by using the ESPRIT (estimation of signal parameters by rotational invariance techniques) method [4] [6]. In our algorithm the complex gains are estimated by using the parametric channel model as in [4] while considering the time variation within one OFDM symbol. More precisely, for a block of OFDM symbols, the complex gains time average over the effective duration of each OFDM symbol of different paths are estimated using LS criteria. After that, the time variation of the different paths complex gains within one OFDM symbol are obtained by using low-pass interpolation. Hence, the channel matrix can be computed and the ICI is reduced by using SIS in data symbol detection. The present proposed algorithm has demonstrated great improvement as compared to the one presented in [10] thanks to an iterative procedure, by estimating and removing the ICI at each iteration. The second contribution of this article is to give a theoretical and simulated Mean Square Error (MSE) channel estimation analysis in function of the normalised (by the OFDM symbol-time) Doppler spread. This further demonstrates the effectiveness of the proposed algorithm.

This paper is organized as follows. Section II introduces the OFDM baseband model and section III multipath complex gains estimation and iterative algorithm. Next, Section IV gives some simulation results. We conclude the paper in Section V.

Notation: Superscripts $(\cdot)^{T}$ and $(\cdot)^{H}$ stand for transpose and Hermitian operators, respectively. $\operatorname{Tr}(\cdot)$ and $E[\cdot]$ are the trace and expectation operations, respectively. $\|\cdot\|$ and $(\cdot)^{*}$ are the magnitude and conjugate of a complex number, respectively. $\_$and $\doteq$ denote a vector and a matrix, respectively. $\mathrm{A}[\mathrm{m}]$ denotes $\overline{\overline{\overline{t h}}} m$ th entry of the vector $\underline{A}$ and $\mathrm{A}[\mathrm{m}, \mathrm{n}]$ denotes the $[m, n]$ th entry of the matrix $\underline{\underline{A}}$.

\section{SYSTEM MODEL}

Suppose that the symbol duration after serial-to-paralllel ( $\mathrm{S} / \mathrm{P})$ conversion is $T_{u}$. The entire signal bandwith is covered by $\mathrm{N}$ subcarriers, and the space between two neighboring subcarriers is $1 / T_{u}$. Denoting the sampling time by $T_{s}=$ $T_{u} / N$, and assuming that the length of the cyclic prefix is 
$T_{g}=N_{g} T_{s}$ with $N_{g}$ being an integer. The duration of an OFDM symbol is $T=\left(N+N_{g}\right) T_{s}$. In an OFDM system, the transmitter usually applies an $N$-point IFFT to data block QAM-symbols $\left\{X_{n}[k]\right\}$, where $\mathrm{n}$ and k represent respectively the OFDM symbol index and the subcarrier index, and adds the cyclic prefix (CP), wich is a copy of the last samples of the IFFT output, to avoid inter-symbol-interference (ISI) caused by multipath fading channels. In order to limit the periodic spectrum of the discrete time signal at the output of the IFFT, we use an appropriate analog transmission filter $G_{e}(f)$. As a result, the output baseband signal of the transmitter can be represented as [4]:

$$
x(t)=\sum_{n=-\infty}^{\infty} \sum_{k=-\frac{N}{2}}^{\frac{N}{2}-1} X_{n}[k] \phi_{k}(t-n T) \otimes g_{e}(t)
$$

where $\otimes$ denotes the convolution, $g_{e}(t)$ is the impulse response of the transmission analog filter, and $\phi_{k}(t)$ is the subcarrier pulse that can be described by:

$$
\phi_{k}(t)= \begin{cases}\frac{1}{T_{u}} e^{j 2 \pi \frac{k}{T_{u}} t} & t \in\left[-T_{g}, T_{u}\right] \\ 0 & \text { otherwise }\end{cases}
$$

It is assumed that the signal is transmitted over a multipath Rayleigh fading channel characterized by:

$$
h(t, \tau)=\sum_{l=1}^{L} \alpha_{l}(t) \delta\left(\tau-\tau_{l} T_{s}\right)
$$

where $L$ is the number of paths, $\alpha_{l}$ is the $l$ th complex gains of variance $\sigma_{\alpha_{l}}^{2}$ and $\tau_{l}$ is the lth delay normalized by the sampling time ( $\tau_{l}$ is not necessarily an integer). $\left\{\alpha_{l}(t)\right\}$ are wide-sense stationary (WSS) narrow-band complex Gaussian processes with the so-called Jakes' power spectrum [5] and uncorellated with respect to each other. The average energy of the channel is normalized to one.

At the receiver side, after passing to discret time through low pass filtering and $A / D$ conversion, the guard time is removed assuming that the $\mathrm{CP}$ length is no less than the maximum delay. Afterwards, a $N$-point FFT is applied to transform the sequence into frequency domain. The $k$ th subcarrier output of FFT during the $n$th OFDM symbol can be represented by:

$$
Y_{n}[k]=\sum_{m=-\frac{N}{2}}^{\frac{N}{2}-1} X_{n}[m] G_{e}[m] G_{r}[m] H_{n}[k, m]+W_{n}[k]
$$

where $W_{n}[k]$ is white complex Gaussian noise with variance $\sigma^{2}, G_{e}[m]$ and $G_{r}[m]$ are the transmitter and receiver filter frequency response values at the $m$ th transmited subcarrier frequency, and $H_{n}[k, m]$ are the coefficients of the channel matrix from $m$ th transmited subcarrier frequency to $k$ th received subcarrier frequency given by:

$$
H_{n}[k, m]=\frac{1}{N} \sum_{l=1}^{L}\left[e^{-j 2 \pi \frac{m}{N} \tau_{l}} \sum_{q=0}^{N-1} \alpha_{l}^{n}\left(q T_{s}\right) e^{j 2 \pi \frac{m-k}{N} q}\right]
$$

where $k, m \in\left[-\frac{N}{2}, \frac{N}{2}-1\right]$ and $\left\{\alpha_{l}^{n}\left(q T_{s}\right)\right\}$ is the $T_{s}$ spaced sampling of $l$ th complex gain during the $n$th OFDM symbol.

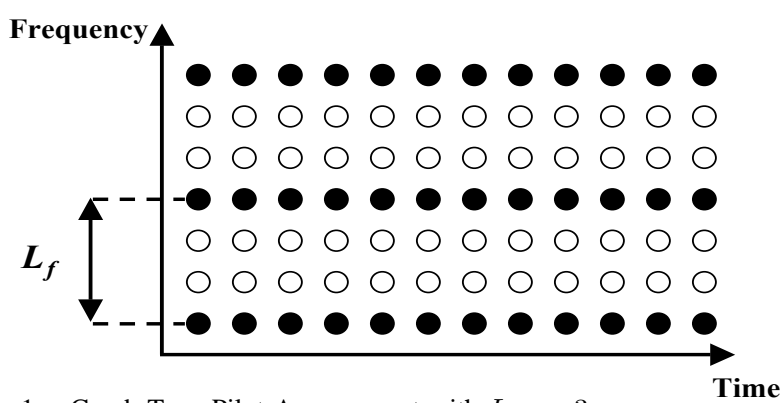

Fig. 1. Comb-Type Pilot Arrangement with $L_{f}=3$

If we assume $N$ transmission subcarriers within the flat region of the transmitter and receiver filter frequency response then by using the matrix notation and omitting the index time $n$, (4) can be rewritten as:

$$
\underline{Y}=\underline{\underline{H}} \underline{X}+\underline{W}
$$

where $G_{e}[m]$ and $G_{r}[m]$ are assumed to be equal to one in the flat region. $\underline{X}, \underline{Y}, \underline{W}$ are $N \times 1$ vectors and $\underline{H}$ is an $N \times N$ channel matrix, which contains the time average of the channel frequency response $H[k, k]$ on its diagonal and the coefficients of the inter-carrier interference (ICI) $H[k, m]$, $k \neq m$, otherwise.

\section{MULTIPATH COMPLEX GAINS ESTIMATION AND ITERATIVE ALGORITHM}

In this section, we propose a method based on comb-type pilots and multipath time delays information to estimate the sampled complex gains $\left\{\alpha_{l}\left(q T_{s}\right)\right\}$ with sampling period $T_{s}$.

\section{A. Pilot Pattern and Received Pilot Subcarriers}

The $N_{p}$ pilot subcarriers are evenly inserted into the $N$ subcarriers as shown in Fig 1 where $L_{f}$ denotes the interval in terms of the number of subcarriers between two adjacent pilots in the frequency domain. $L_{f}$ can be selected without the need for respecting the sampling theorem (in frequency domain) as opposed to the methods shown in [4] [3]. However, as we will see with equation (12), $N_{p}$ must fulfill the following requirement: $N_{p} \geq L$.

Let $\mathcal{P}$ denote the set that contains the index positions of the $N_{p}$ pilot subcarriers. Then

$$
\mathcal{P}=\left\{p_{s} \mid p_{s}=s L_{f}-\frac{N}{2}, s=0, \ldots, N_{p}-1\right\}
$$

The received pilot subcarriers can be written as the sum of three components:

$$
\underline{Y_{p}}=\underline{\underline{X_{p}}} \underline{H_{p}}+\underline{\underline{H_{p_{I}}}} \underline{X}+\underline{W_{p}}
$$

where $X_{p}$ is an $N_{p} \times N_{p}$ diagonal matrix, $Y_{p}, W_{p}$ are $N_{p} \times 1$ vectors $\overline{\overline{\text { given }}}$ by:

$$
\begin{aligned}
\underline{\underline{X_{p}}} & =\operatorname{diag}\left\{X\left[p_{0}\right], X\left[p_{1}\right], \ldots, X\left[p_{N_{p}-1}\right]\right\} \\
\underline{Y_{p}} & =\left[Y\left[p_{0}\right], Y\left[p_{1}\right], \ldots, Y\left[p_{N_{p}-1}\right]\right]^{T} \\
\underline{W_{p}} & =\left[W\left[p_{0}\right], W\left[p_{1}\right], \ldots, W\left[p_{N_{p}-1}\right]\right]^{T}
\end{aligned}
$$




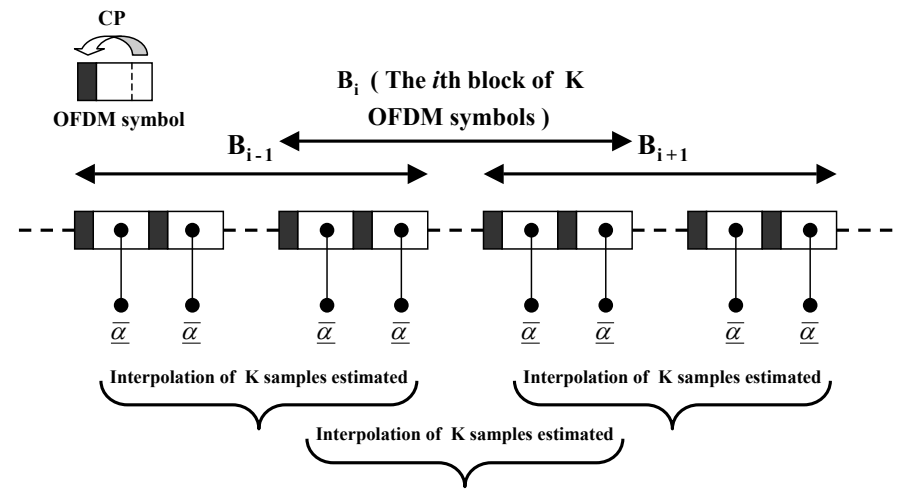

Fig. 2. The diagram of complex gains estimator

$\underline{H_{p}}$ is an $N_{p} \times 1$ vector and $\underline{H_{p_{I}}}$ is an $N_{p} \times N$ matrix with

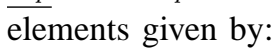

$$
\begin{aligned}
H_{p}\left[p_{s}\right] & =H\left[p_{s}, p_{s}\right]=\sum_{l=1}^{L} \overline{\alpha_{l}} e^{-j 2 \pi \frac{p_{s}}{N} \tau_{l}} \\
H_{p_{I}}\left[p_{s}, m\right] & = \begin{cases}H\left[p_{s}, m\right] & \text { if } m \in\left[-\frac{N}{2}, \frac{N}{2}-1\right]-\mathcal{P} \\
0 & \text { if } m \in \mathcal{P}\end{cases} \\
\text { with } \overline{\alpha_{l}} & =\frac{1}{N} \sum_{q=0}^{N-1} \alpha_{l}\left(q T_{s}\right)
\end{aligned}
$$

$\overline{\alpha_{l}}$ is the time average over the effective duration of OFDM symbol of the $l$ th complex gain. The first component is the desired term without ICI and the second component is the ICI term. $H_{p}$ can be writen as the Fourier transform for the different complex gains time average $\left\{\overline{\alpha_{l}}\right\}$ :

$$
\underline{H_{p}}=\underline{\underline{F_{p}}} \underline{\bar{\alpha}}
$$

where $F_{p}$ and $\underline{\bar{\alpha}}$ are respectively the $N_{p} \times L$ Fourier transform matrix $\overline{\overline{\text { and }}}$ d the $N_{p} \times 1$ vector given by:

$$
\underline{\underline{F_{p}}}=\left[\begin{array}{ccc}
e^{-j 2 \pi \frac{p_{0}}{N} \tau_{1}} & \cdots & e^{-j 2 \pi \frac{p_{0}}{N} \tau_{L}} \\
\vdots & \ddots & \vdots \\
e^{-j 2 \pi \frac{p_{N_{p}-1}}{N} \tau_{1}} & \cdots & e^{-j 2 \pi \frac{p_{N_{p}-1}}{N} \tau_{L}}
\end{array}\right]
$$

\section{B. Estimation of Multipath Complex Gains}

The complex gains time averages over the effective duration of each OFDM symbol for the different paths are estimated using the LS criteria. By neglecting the ICI contribution, the LS-estimator of $\bar{\alpha}$ is:

$$
\begin{aligned}
\underline{\bar{\alpha}}_{L S} & =\underline{\underline{M}} \underline{Y_{p}} \\
\text { with } \underline{\underline{M}} & =\left(\underline{\underline{F_{p}}} \underline{\underline{X_{p}}}{ }^{H} \underline{\underline{X_{p}}} \underline{\underline{F_{p}}}\right)^{-1} \underline{\underline{F_{p}}} \underline{\underline{\underline{X_{p}}}}
\end{aligned}
$$

Since the $T_{s}$-spaced sampling of the complex gain taken in the middle of the effective duration of OFDM symbol is closest to the complex gain time average over the effective duration, then we assume that $\underline{\bar{\alpha}}_{L S}$ is an estimation of $\underline{\alpha}_{c}=\left[\alpha_{1}\left(\frac{N}{2} T_{s}\right), \ldots, \alpha_{L}\left(\frac{N}{2} T_{s}\right)\right]^{T}$. So, by estimating $\underline{\alpha}$ for some OFDM symbols and interpolating them by a factor $\left(N+N_{g}\right)$ by using low-pass interpolation [3], we obtain an estimation of the sampled complex gains $\left\{\alpha_{l}\left(q T_{s}\right)\right\}$ at time $T_{s}$ during these OFDM symbols, for each path.

\section{Iterative Algorithm}

In the iterative algorithm of channel estimation and ICI suppression, the OFDM symbols are grouped in blocks of $K$ OFDM symbols each one. Each two consecutive blocks are intersected in two OFDM symbols as shown in Fig 2. For a block of $K$ OFDM symbols, the iterative algorithm proceeds as following:

$$
\begin{aligned}
& \text { initialization: } \\
& i \leftarrow 1 \\
& \underline{Y_{p}}{ }^{k, i}=\underline{Y_{p}}{ }^{k}
\end{aligned}
$$

\section{recursion:}
1) $\underline{\bar{\alpha}}_{L S}^{k, i}=\underline{\underline{M}} \underline{Y}^{k, i}$
2) $\left\{\hat{\alpha}_{l}^{k, i}\left(q \overline{T_{s}}\right), \quad \begin{array}{l}k=2, \ldots, K-1 \\ q=-N_{g}, \ldots, N-1\end{array}\right\}=\operatorname{interp}\left(\bar{\alpha}_{l_{L S}, i}^{k, i}, N+N_{g}\right)$
3) compute using (5) the channel matrix $\underline{\hat{H}}^{k, i}$
4) remove the ICI of pilots from the received data subcarriers $Y_{d}^{k}$
5) detection of data symbols $\hat{X}_{d}^{k, i}$
6) $\underline{Y p}^{k, i+1}=\underline{Y}^{k}-\hat{H}_{p_{I}}^{k, i} \underline{\hat{X}}^{k, i}$
7) $i \leftarrow i+1$

where interp denotes the interpolation function and, $i$ and $k$ represent the iteration number and the number of OFDM symbol in a block, respectively. Note that, the steps 3 to 6 are executed without considering the first and the last OFDM symbols (i.e., $k=2$ to $K-1$ ) in order to avoid limiting effects of interpolation. The data symbols detection in step 5 are estimated by successive data interference suppresion (SIS) scheme with the optimal ordering and one tap frequency equalizer [10].

\section{Mean Square Error (MSE) Analysis}

The MSE of the LS-estimator of $\underline{\alpha}$ is given by:

$$
\begin{gathered}
m_{s e_{1}}=E\left[\left(\underline{\bar{\alpha}}_{L S}-\underline{\bar{\alpha}}\right)^{H}\left(\underline{\bar{\alpha}}_{L S}-\underline{\bar{\alpha}}\right)\right]=\operatorname{Tr}\left(\underline{\underline{M}} \underline{\underline{R}} \underline{\underline{M}}^{H}\right) \\
\text { with } \underline{\underline{R}}=E\left[\underline{\underline{H_{p_{I}}}} \underline{X} \underline{X}^{H} \underline{\underline{H_{p_{I}}}}{ }^{H}\right]+\sigma^{2} \underline{\underline{I}}_{N_{p}}
\end{gathered}
$$

It is clear that our LS-estimator is unbiased. So, the CRAMERRAO BOUND (CRB) [11] is an important criteria to evaluate how good our LS-estimator can be since it provides the MMSE bound among all unbiased estimators. The Standard CRB (SCRB) for the estimator of $\underline{\bar{\alpha}}$ with ICI known is given by:

$$
\operatorname{SCRB}(\underline{\bar{\alpha}})=\frac{1}{S N R} \operatorname{Tr}\left(\left(\underline{\underline{F_{p}}} \underline{\underline{X_{p}}}{ }^{H} \underline{\underline{X_{p}}} \underline{\underline{F_{p}}}\right)^{-1}\right)
$$

where $S N R$ is the normalized signal to noise ratio. It is easy to show that

$$
\left\{\begin{array}{l}
\operatorname{mse}_{1}(\text { with ICI })>S C R B(\underline{\bar{\alpha}}) \\
\left.m e_{1} \text { (without ICI }\right)=S C R B(\underline{\bar{\alpha}})
\end{array}\right.
$$

So, by iteratively estimating and removing the ICI $m s e_{1}$ will be closer to $S C R B(\underline{\bar{\alpha}})$.

The MSE of the assumption that $\underline{\bar{\alpha}}_{L S}$ is an estimation of $\underline{\alpha}_{c}$ is given by:

$$
\begin{aligned}
m s e_{c} & =E\left[\left(\underline{\bar{\alpha}}_{L S}-\underline{\alpha}_{c}\right)^{H}\left(\underline{\bar{\alpha}}_{L S}-\underline{\alpha}_{c}\right)\right] \\
& =m s e_{1}+m s e_{2}+m s e_{12}+m s e_{21}
\end{aligned}
$$


where $m^{2} e_{2}$ is the MSE between $\underline{\bar{\alpha}}$ and $\underline{\alpha}_{c}$ and, $m s e_{12}$ and $m_{s e} 1$ are the cross-covariance terms, which are very negligible, given by:

$$
\begin{aligned}
m s e_{2}= & E\left[\left(\underline{\bar{\alpha}}-\underline{\alpha}_{c}\right)^{H}\left(\underline{\bar{\alpha}}-\underline{\alpha}_{c}\right)\right] \\
= & \sum_{l=1}^{L} \sigma_{\alpha_{l}}^{2}\left(\frac{1}{N^{2}} \sum_{q_{1}=0}^{N-1} \sum_{q_{2}=0}^{N-1} J_{0}\left(2 \pi f_{d} T_{s}\left(q_{1}-q_{2}\right)\right)\right. \\
& \left.-\frac{2}{N} \sum_{q=0}^{N-1} J_{0}\left(2 \pi f_{d} T_{s}\left(q-\frac{N}{2}\right)\right)+1\right) \\
m_{12}= & E\left[(\underline{\bar{\alpha}} L S-\underline{\bar{\alpha}})^{H}\left(\underline{\bar{\alpha}}-\underline{\alpha}_{c}\right)\right] \\
= & \operatorname{Tr}\left(E\left[\left(\underline{\bar{\alpha}}-\underline{\alpha}_{c}\right) \underline{X}^{H}{\underline{\underline{p_{I}}}}^{H}\right] \underline{\underline{M}}^{H}\right) \\
m_{21}= & E\left[\left(\underline{\bar{\alpha}}-\underline{\alpha}_{c}\right)^{H}\left(\underline{\bar{\alpha}}_{L S}-\underline{\bar{\alpha}}\right)\right]=m s e_{12}^{*}
\end{aligned}
$$

where $J_{0}(\cdot)$ denotes the zeroth-order Bessel function of the first kind and $f_{d}$ is the channel maximum Doppler frequency.

The MSE of the multipath complex gain estimator at time $T_{s}$ is given by:

$$
\begin{gathered}
\text { mse }_{T_{s}}=\sum_{k=2}^{K-1} \sum_{q=-N_{g}}^{N-1} E\left[\left(\underline{\hat{\alpha}}_{q}^{k}-\underline{\alpha}_{q}^{k}\right)^{H}\left(\underline{\hat{\alpha}}_{q}^{k}-\underline{\alpha}_{q}^{k}\right)\right] \\
\text { with } \underline{\alpha}_{q}^{k}=\left[\alpha_{1}^{k}\left(q T_{s}\right), \ldots, \alpha_{L}^{k}\left(q T_{s}\right)\right]^{T}
\end{gathered}
$$

Assuming performant interpolator and respecting sampling theorem in time domain $\left(f_{d} T \leq 0.5\right)$, then we have:

$$
m s e_{T_{s}} \approx m s e_{c}
$$

\section{SIMULATION RESULTS}

In this section, we verify the theory by simulation and we test the performance of the iterative algorithm. The mean square error (MSE) and the bit error rate (BER) performances in terms of the average signal-to-noise ratio (SNR) [4] [3] and maximum Doppler spread $f_{d} T$ (normalized by $1 / T$ ) for Rayleigh channel are examined. The normalized channel model is Rayleigh as recommended by GSM Recommendations 05.05 [7] [9], with parameters shown in the table below $\left(\frac{1}{T_{s}}=2 \mathrm{MHz}\right)$. A 4QAM-OFDM system with normalized symbols, $N=128$ subcarriers, $N_{g}=\frac{N}{8}$ subcarriers, $N_{p}=16$ pilots (i.e., $L_{f}=8$ ) and $K=10$ OFDM symbols in each block is used. (note that $(S N R) d B=\left(\frac{E_{b}}{N_{0}}\right) d B+3 d B$ ). These parameters are selected in order to be in concordance with the standard Wimax IEEE802.16e (spacing between subcarriers must be about $10 \mathrm{KHz}$ for a carrier frequency $f_{c}=2.5 \mathrm{GHz}$ ). The BER performance of our iterative algorithm is evaluated under a rapid time-varying channel such as $f_{d} T=0.05$ and $f_{d} T=0.1$ corresponding to a vehicle speed $V_{m}=140 \mathrm{~km} / \mathrm{h}$ and $V_{m}=280 \mathrm{~km} / \mathrm{h}$, respectively, for $f_{c}=5 \mathrm{GHz}$.

\begin{tabular}{|c|c|c|}
\hline \multicolumn{3}{|c|}{ Rayleigh Channel } \\
\hline Path Number & Average Power $(d B)$ & Normalized Delay \\
\hline 1 & -7.219 & 0 \\
\hline 2 & -4.219 & 0.4 \\
\hline 3 & -6.219 & 1 \\
\hline 4 & -10.219 & 3.2 \\
\hline 5 & -12.219 & 4.6 \\
\hline 6 & -14.219 & 10 \\
\hline
\end{tabular}

Fig 3 shows the MSE in terms of $f_{d} T$ for $S N R=20 d B$. It is observed that, with all ICI, the MSE obtained by simulation
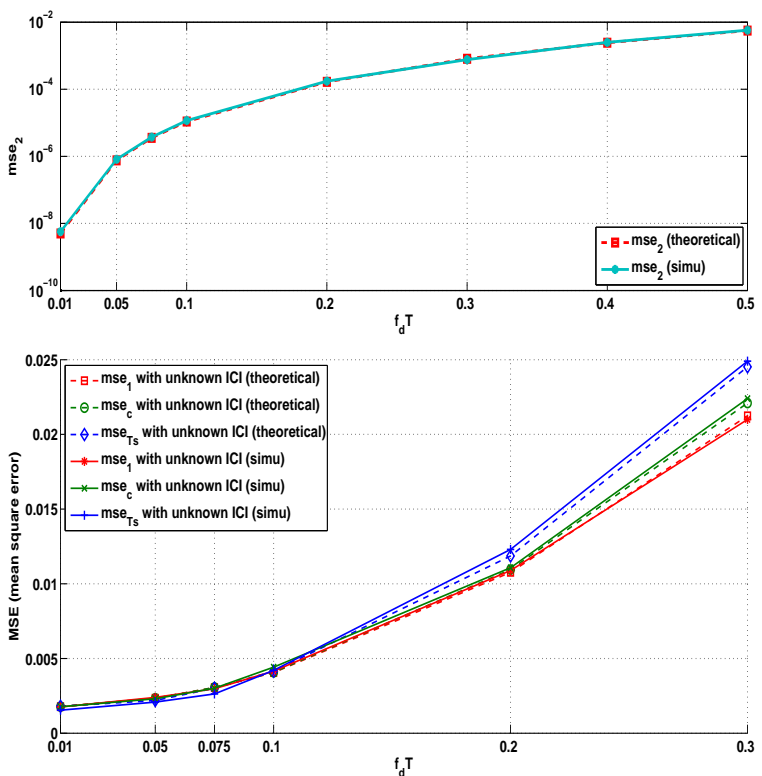

Fig. 3. Comparison between mean square errors (MSE) for $S N R=20 d B$

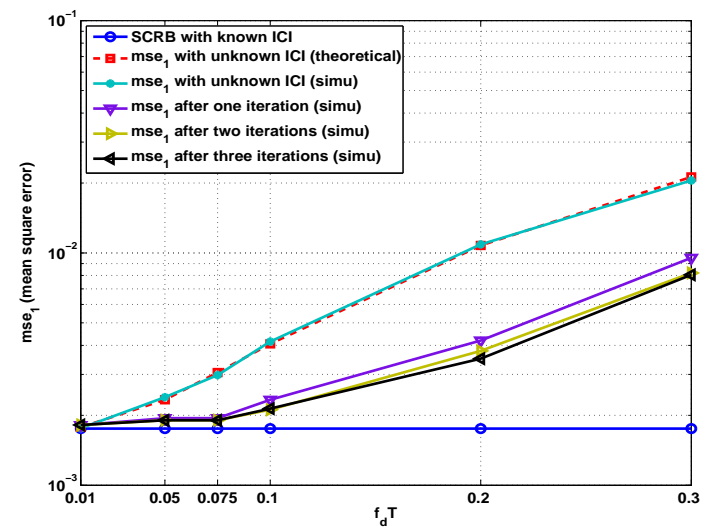

Fig. 4. The mean square error of the LS-estimator for $S N R=20 d B$

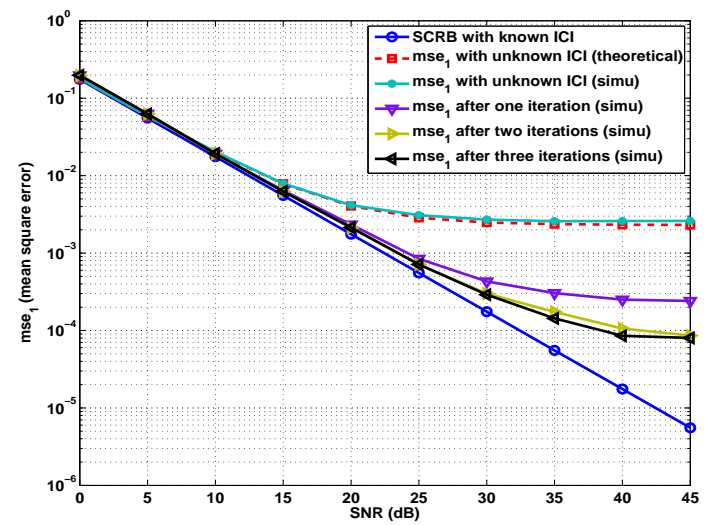

Fig. 5. The mean square error of the LS-estimator for $f_{d} T=0.1$

verify the theoretical MSE. We verify that $m s e_{T_{s}} \approx m s e_{c}$, that means our interpolation method over a block of $K$ OFDM symbols is adequate. For $f_{d} T \leq 0.1, m_{s e}$ is negligible with respect to $m s e_{1}$, thus $m s e_{T_{s}} \approx m s e_{1}$.

Fig 4 gives the evolution of $m s e_{1}$ with the iterations in terms of $f_{d} T$ for $S N R=20 d B$. We notice that, with all ICI, $m_{s e}$ is far from SCRB and when we commence to reduce the ICI, by improving the estimation of ICI at each iteration, $m s e_{1}$ shows a significant improvement especially after the first 


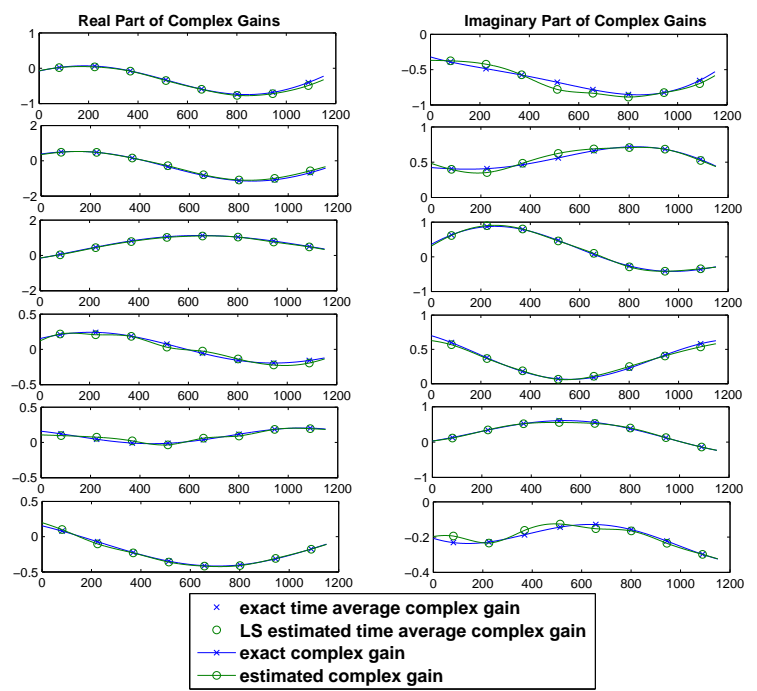

Fig. 6. The LS estimated complex gain of six paths over 8 OFDM symbols after one iteration with $S N R=20 d B$ and $f_{d} T=0.1$

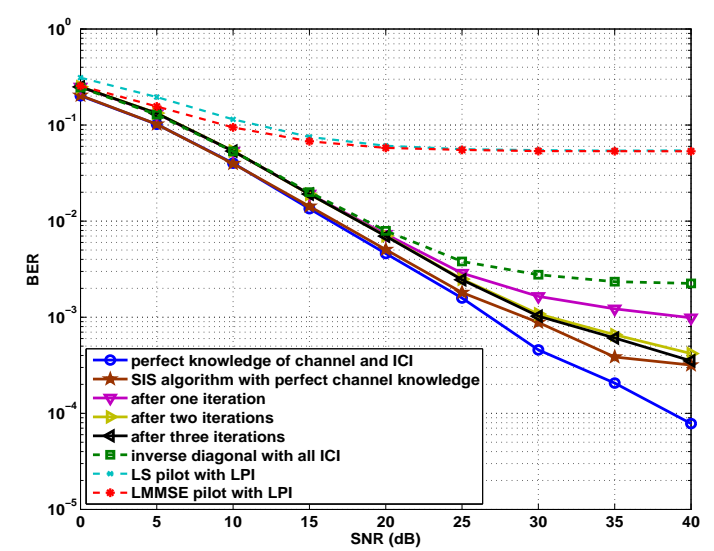

Fig. 7. Comparison of BER for $f_{d} T=0.05$

iteration and approaches the SCRB for $f_{d} T \leq 0.1$. However, by increasing $f_{d} T$, we show from $\mathrm{mse}_{2}$ given in Fig 3 that $\underline{\bar{\alpha}}$ moves away from $\underline{\alpha}_{c}$. Hence, for $f_{d} T>0.1$, MSE of multipath complex gains estimator is significant and the ICI are not estimated and nor removed perfectly.

Fig 5 shows the evolution of $m s e_{T_{s}} \approx m s e_{1}$ with the iterations in terms of $S N R$ for $f_{d} T=0.1$. After one iteration, a great improvement is realized and $m s e_{1}$ is very close to SCRB especially in low and moderate SNR region. This is because for low SNR the noise is dominant relative to the ICI level and, for high SNR ICI are not completely removed due to the data symbols detection error.

For illustration, Fig 6 illustrates the real and the imaginary part of the exact and estimated (after one iteration) multipath complex gain for one channel realization over 8 OFDM symbols with $S N R=20 d B$ and $f_{d} T=0.1$. It is well observed that we have a good estimation of multipath complex gains for very rapidly channel.

Fig 7 and Fig 8 gives the BER performance of our proposed iterative algorithm, compared to conventional methods (LS and LMMSE criteria with LPI in frequency domain) [2] [3] and SIS algorithm with perfect channel knowledge for $f_{d} T=0.05$ and $f_{d} T=0.1$. We plotted also as reference, performance obtained with perfect knowledge of channel and ICI. This result shows that, with all ICI, our algorithm performs better than

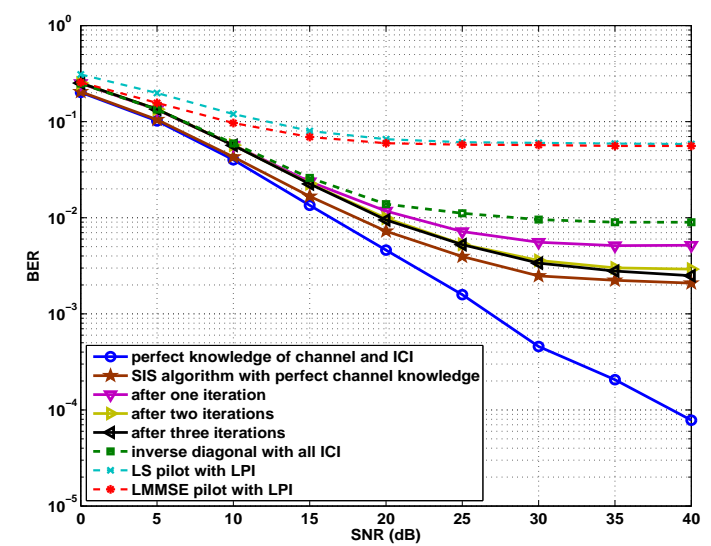

Fig. 8. Comparison of BER for $f_{d} T=0.1$

the conventional methods. Moreover, when we start removing ICI our iterative algorithm offers an improvement in BER after each iteration because the estimation of ICI is improved during each iteration. After two iterations, performance of our algorithm and SIS algorithm with perfect channel knowledge are very close. At high SNR, we cannot reach the performance obtained with perfect knowledge of channel and ICI because we have an error floor due to data symbols detection error.

\section{CONCLUSION}

In this paper, we have presented an iterative algorithm to estimate multipath complex gains and mitigate the intersub-carrier-interference (ICI) for OFDM system. Theoretical analysis and simultion results of our iterative algorithm show that, by estimating and removing the ICI at each iteration, multipath complex gains estimation and coherent demodulation have a great improvement especially after the first iteration for high Doppler spread.

\section{REFERENCES}

[1] A. R. S. Bahai and B. R. Saltzberg, Multi-Carrier Dications: Theory and Applications of OFDM: Kluwer Academic/Plenum, 1999.

[2] M. Hsieh and C. Wei, "Channel estimation for OFDM systems based on comb-type pilot arrangement in frequency selective fading channels" in IEEE Trans. Consumer Electron., vol.44, no. 1, Feb. 1998.

[3] S. Coleri, M. Ergen, A. Puri and A. Bahai, "Channel estimation techniques based on pilot arrangement in OFDM systems" in IEEE Trans. Broad., vol. 48. no. 3, pp. 223-229 Sep. 2002.

[4] B. Yang, K. B. Letaief, R. S. Cheng and Z. Cao, "Channel estimation for OFDM transmisson in mutipath fading channels based on parametric channel modeling" in IEEE Trans. Commun., vol. 49, no. 3, pp. 467-479, March 2001.

[5] W. C. Jakes, Microwave Mobile Communications. Piscataway, NJ: IEEE Press, 1983.

[6] R. Roy and T. Kailath, "ESPRIT-Estimation of signal parameters via rotational invariance techniques" in IEEE Trans. Acoust., Speech, Signal Processing, vol. 37, pp. 984-995, July 1989.

[7] European Telecommunications Standards Institute, European Digital Cellular Telecommunication System (Phase 2); Radio Transmission and Reception, GSM 05.05, vers. 4.6.0, Sophia Antipolis, France, July 1993.

[8] E. Simon, L. Ros and K. Raoof," Synchronization over rapidly timevarying multipath channel for CDMA downlink RAKE receivers in TimeDivision mode", in IEEE Trans. Vehicular Techno., to be appeared.

[9] Y. Zahao and A. Huang, "A novel Channel estimation method for OFDM Mobile Communications Systems based on pilot signals and transform domain processing" in Proc. IEEE 47th Vehicular Techno. Conf., Phonix, USA, May 1997, pp. 2089-2093.

[10] H. Hijazi, L. Ros and G. Jourdain, “ OFDM Channel Parameters Estimation used for ICI Reduction in time-varying Multipath channels" in EUROPEAN WIRELESS Conf., Paris, FRANCE, April 2007.

[11] H. L. Van Trees, Detection, estimation, and modulation theory: Part I, Wiley, New York, 1968. 